\title{
A new 3D finite element for the finite deformation of nearly incompressible hyperelastic solids
}

\author{
Ashutosh Bijalwan and B.P. Patel* \\ Department of Applied Mechanics, \\ Indian Institute of Technology Delhi, \\ New Delhi-110016, India \\ Email: ashutoshbijalwan48@gmail.com \\ Email: bppatel@am.iitd.ac.in \\ *Corresponding author
}

\begin{abstract}
Volumetric locking is exhibited by nearly incompressible solids such as rubber, resulting in over-stiffening response of the finite element mesh. In this work, we developed the displacement-based computationally efficient volumetric locking-free 3D finite element using smoothening of determinant of deformation gradient (J-Bar method) within the framework of isotropic hyperelasticity. The developed methodology is employed to analyse a rubber block undergoing finite stretch and bending deformations. The convergence study for finite stretch and bending of rubber block is presented. Results of the analysis show that J-Bar method efficiently removes the volumetric locking.
\end{abstract}

Keywords: incompressible; locking; finite deformation; J-Bar method; Neo-Hookean rubber.

Reference to this paper should be made as follows: Bijalwan, A. and Patel, B.P. (2019) 'A new 3D finite element for the finite deformation of nearly incompressible hyperelastic solids', Int. J. Materials and Structural Integrity, Vol. 13 , Nos. 1/2/3, pp.67-80.

Biographical notes: Ashutosh Bijalwan completed his Bachelor's in Mechanical Engineering from the Govind Ballabh Pant University of Agriculture and Technology Pantnagar, India and Master's in Engineering Mechanics from the department of Applied Mechanics, Indian Institute of Technology Delhi, India. His research interests include finite element analysis and nonlinear mechanics of solids.

B.P. Patel is a Professor of Applied Mechanics, Indian Institute of Technology Delhi, India. He received his PhD in Applied Mechanics from the Motilal Nehru National Institute of Technology Allahabad, India. His research interests include finite element method, nonlinear dynamics, continuum damage mechanics, smart structures, composite mechanics and multi-scale modelling of nanostructures.

This paper is a revised and expanded version of a paper entitled "Volumetric locking free 3D finite element for nearly incompressible hyperelastic solids' presented at 3rd Indian Conference on Applied Mechanics (INCAM2017), Motilal Nehru National Institute of Technology Allahabad, Allahabad, India, 5-7 July 2017. 


\section{Introduction}

In industrial sector, rubber is used for shock mounts, gaskets and seals, hose and tubing undergoing finite deformation. These applications are critical and require an adequate understanding of nonlinear and isochoric behaviour of rubber. To model rubber-like nearly incompressible solids, neo-Hookean, Mooney-Rivlin, Ogden, and Yeoh models are some of the commonly used models (Boyce and Arruda, 2000; Guo and Sluys, 2006). Finite element method is one of the most popular tools for analysis of complex problems (Liu et al., 2016).

Similar to rubber, polymers, biological tissues (for example, ligaments and liver) also exhibit nearly isochoric behaviour and undergo finite deformation in their service life. The main challenging aspect in the finite element modelling of such materials is their nearly incompressible behaviour. Incompressibility constraint imposes severe restriction on the displacement field resulting in the over stiffening response of the finite element mesh and is termed as volumetric locking. Volumetric locking in nearly incompressible solids is a current field of research in solid mechanics (Elguedj et al., 2008a; Wriggers and Carstensen, 2009; Bernardin et al., 2015a; Foster and Nejad, 2015). Many techniques have been developed to avoid volumetric locking viz. selective reduced integration (SRI), mixed formulation, B-Bar method and F-Bar method (Wriggers and Carstensen, 2009) each one with their own merits and demerits. Although SRI scheme can suppress volumetric locking and is also quite simple for implementation (Malkus and Hughes, 1978), however, it may lead to the ill conditioning of stiffness matrix resulting in the excitation of hourglass modes (Fried, 1974; Hughes, 1987). The multiplicative decomposition of deformation gradient into dilatational and deviatoric parts leads to the development of mixed formulation, B-Bar and F-Bar methods (Flory, 1961). Akhrass et al. (2012) explored displacement-pressure ( $u-p)$ mixed formulation for modelling finite deformation of incompressible hyperelastic solids using subgrid scale approach. Suzuki and Bittencourt (2013) developed hp-FEM-based element by utilising the u-p mixed formulation with pressure projection technique to study the Mullins effect in isotropic solids. Based on the multiplicative decomposition of deformation gradient, Simo et al. (1985) proposed three field mixed formulation to counter the volumetric locking. Weiss et al. (1996) applied three field principle to study the hyperelastic behaviour of ligament whereas Holzapfel (1996) studied the viscohyperelastic response of rubber using Ogden model. Main challenges reside in the added computational complexities due to the introduction of additional field variables viz. hydrostatic pressure and Jacobian of deformation gradient. In B-Bar method, strain displacement matrix is split into dilatational and deviatoric parts (Hughes, 1980). Foster and Nejad (2015) developed a volumetric locking free element based on the volumetric average of Jacobian within the framework of B-Bar method. F-Bar method of de Souza Neto et al. (1996) assumed Jacobian as a constant evaluated at the centre of the element whereas the mean dilatational approach of Nagtegaal et al. (1974) evaluated the Jacobian as the volume average within the element. Recently, Elguedj et al. (2008b) and Bernardin et al. (2015b) have applied an alternative locking-free approach based on the volumetric-average nodal projection (VANP) method for nearly incompressible hyperelastic solids.

From the detailed literature review, it is found that in the F-Bar/B-Bar methods, the deformation gradient $(\mathbf{F})$ is replaced by a modified deformation gradient $(\overline{\mathbf{F}})$ in both the deviatoric and dilatational parts of energy, making the linearisation process complex and 
computationally expensive. It may be noted that the locking manifests only from the dilatational part of strain energy. In view of this, current work proposes a locking-free element by modifying the dilatational energy term only through J-Bar method, thus retaining the simplicity and computational efficiency of standard displacement-based formulation. In the present study, we have considered neo-Hookean solid undergoing finite deformation, although the developed element is equally applicable to other material models as well.

The paper is organised as follows: first, brief introduction to isotropic hyperelastic constitutive model is given in Section 2. Then in Section 3, finite element formulation is presented and new locking-free element is introduced. Implementation algorithm of both standard and proposed locking-free scheme is described in Section 4. To demonstrate the robustness of the developed locking-free element, numerical simulations based on eight noded and 27 noded brick elements are presented in Section 5. Finally, the concluding remarks are made in Section 6.

\section{Isotropic hyperelastic constitutive model}

Let $x$ be the deformation mapping of each material point $X$ in the reference configuration to the corresponding point in the deformed configuration. Deformation gradient $(F)$, right Cauchy-Green tensor $(\mathbf{C})$ and Green-Lagrange strain tensor $(\mathbf{E})$ are defined as $\mathbf{F}=\frac{d x}{d X}$, $\mathbf{C}=\mathrm{F}^{\mathrm{T}} \mathrm{F}, \mathbf{E}=\frac{1}{2}(\mathbf{C}-\mathrm{I})$, respectively, where $\mathrm{I}$ is the second rank isotropic tensor. Furthermore, deformation is such that it does not violate the homeomorphism of the bodies and deformed shape, i.e., det $(F) \geq 0$. For simple elastic material undergoing isothermal deformation, Clausius-Duhem inequality ensures that the internal dissipation is always greater than or equal to zero (Bertram, 2007), i.e.

$$
\mathcal{D}_{\text {int }}:=\left(\mathrm{S}-\frac{\partial \Psi}{\partial \mathrm{E}}\right): \dot{\mathrm{E}}-\sum_{k=1}^{n} \frac{\partial \Psi}{\partial Q^{k}}: \dot{Q}^{k} \geq 0
$$

where $\Psi$ is Helmholtz free energy per unit reference volume, $S$ is second Piola-Kirchhoff stress tensor and $Q^{k}$ is stress like internal variable governing the irreversible changes in the evolution of the system. As per Noll-Coleman procedure, material possessing a constitutive relation of the form $\mathbf{S}=\frac{\partial \psi}{\partial \mathbf{E}}$ and $-\frac{\partial \Psi}{\partial Q^{k}}: \dot{Q}^{k} \geq 0$ for all admissible processes is called a hyperelastic material.

Strain energy of isotropic hyperelastic material must be invariant under rigid body rotation and can be written as a function of invariants of right Cauchy-Green tensor $\left(I_{1}, I_{2}\right.$, $\left.I_{3}\right)$, i.e.

$$
\Psi=\Psi(\mathrm{E})=\Psi\left(\mathbb{Q}^{\mathrm{T}} \mathrm{E} \mathbb{Q}\right)=\Psi\left(I_{1}, I_{2}, I_{3}\right)
$$

where $\square$ is proper orthogonal tensor and invariants are defined as: 


$$
I_{1}=\operatorname{tr}(\mathbf{C}), I_{2}=\frac{1}{2}\left([\operatorname{tr}(\mathbf{C})]^{2}-\operatorname{tr} \mathbf{C}^{2}\right) \text { and } I_{3}=\operatorname{det}(\mathbf{C})=J^{2}
$$

For isotropic hyperelasticity, strain energy decoupled into dilatational and deviatoric parts can be written as a function of invariants of the modified right Cauchy-Green tensor $(\overline{\mathbf{C}})$.

$$
\Psi\left(I_{1}, I_{2}, I_{3}\right)=\Psi_{v o l}(J)+\Psi_{i s o}\left(\bar{I}_{1}, \bar{I}_{2}\right)
$$

where invariants of the modified Cauchy-Green tensor are given by $\bar{I}_{1}=J^{-\frac{2}{3}} I_{1}$ and $\bar{I}_{2}=J^{-\frac{4}{3}} I_{2}$.

Splitting of strain energy leads to the additive split of Second Piola-Kirchhoff stress tensor given by

$$
S=S_{v o l}+S_{i s o}
$$

where $\left(S_{v o l}\right)_{i j}=\frac{\partial \Psi_{v o l}}{\partial J} \frac{\partial J}{\partial E_{i j}}$ and $\left(S_{i s o}\right)_{i j}=\frac{\partial \Psi_{i s o}}{\partial \bar{I}_{1}} \frac{\partial \bar{I}_{1}}{\partial E_{i j}}+\frac{\partial \Psi_{i s o}}{\partial \bar{I}_{2}} \frac{\partial \bar{I}_{2}}{\partial E_{i j}}$

Similarly, constitutive tensor $\left(\square=\frac{\partial \mathrm{S}}{\partial \mathrm{E}}\right)$ can be decomposed as

$$
\square=\square_{\text {vol }}+\square_{\text {iso }}
$$

where $\left(\square_{v o l}\right)_{i j k l}=\frac{\partial\left(S_{v o l}\right)_{i j}}{\partial E_{k l}}$ and $\left(\square_{i s o}\right)_{i j k l}=\frac{\partial\left(S_{i s o}\right)_{i j}}{\partial E_{k l}}$

To ensure uniqueness of boundary value problem (BVP), the strain energy density function should satisfy polyconvexity criterion. Isotropic neo-Hookean model satisfies polyconvexity which in turn guarantees material stability (Schröder and Neff, 2003; Duong et al., 2015). For neo-Hookean solids, deviatoric strain energy is given by: $\Psi_{i s o}\left(\bar{I}_{1}\right)=\frac{c_{1}}{2}\left(\bar{I}_{1}-3\right)$ and volumetric strain energy is given by: $\Psi_{v o l}(J)=\frac{K}{2}(J-1)^{2}$, where $c_{1}$ and $K$ are the shear and bulk modulus, respectively.

\section{Total Lagrangian formulation and J-Bar method}

Consider a 3D domain $\Omega_{o}$ bounded by the surface $\Gamma$. Assuming the boundary conditions are such that the displacements are prescribed over the surface $\Gamma_{u}$ and the tractions over surface $\Gamma_{o}$. The distribution of essential and natural boundary conditions on $\Gamma$ is such that $\Gamma_{u}, \Gamma_{o} \in \Gamma$ and $\Gamma_{u} \cap \Gamma_{o}=\Phi$. Neglecting inertia effects, strong form of the boundary value problem (BVP) is given by

$$
\begin{aligned}
& \nabla(\mathrm{FS})+f^{b}=0 \\
& u=\bar{u} \text { on } \Gamma_{u}
\end{aligned}
$$




$$
\mathrm{P} \dddot{\mathrm{N}}=\mathrm{FS} \dddot{\mathrm{N}}=f^{s} \text { on } \Gamma_{o}
$$

where $f^{b}$ and $f^{s}$ are body and surface forces, respectively, $\mathrm{P}$ is first Piola-Kirchhoff stress tensor and $\dddot{\mathrm{N}}$ is unit outer normal on $\Gamma_{o}$.

Principle of virtual work gives the following weak form of the momentum equation (sum over repeated indices)

$$
\int_{\Omega_{o}} S_{i j} \delta E_{i j} d \Omega_{o}=\int_{\Omega_{o}} f_{i}^{b} \delta u_{i} d \Omega_{o}+\int_{\Gamma_{o}} f_{i}^{s} \delta u_{i} d \Gamma_{o}
$$

Finite element discretisation of weak form is given by

$$
\int_{\Omega_{e}} \delta \tilde{u}_{e}^{T}[B]^{T} \hat{S} d \Omega_{e}-\int_{\Omega_{e}} \delta \tilde{u}_{e}^{T}[N]^{T} f^{b} d \Omega_{e}-\int_{\Gamma_{e}} \delta \tilde{u}_{e}^{T}[N]^{T} f^{s} d \Gamma_{e}=0
$$

where $\tilde{u}_{e}$ is the vector of elemental degrees of freedom, $N$ is shape function matrix, $B$ is strain-displacement matrix and $\hat{S}=\left[S_{11} S_{22} S_{33} S_{12} S_{23} S_{13}\right]^{T}$ is stress vector.

To solve the nonlinear BVP, Newton-Raphson method is employed. Linearising the weak form, we have the following incremental equation

$$
\int_{\Omega_{e}} \frac{\Delta S_{i j}}{\Delta E_{k l}} \delta E_{i j} \Delta E_{k l} d \Omega_{e}+\int_{\Omega_{e}} S_{i j} \delta \Delta E_{i j} d \Omega_{e}=\int_{\Omega_{e}} f_{i}^{b} \delta \Delta u_{i j} d \Omega_{e}+\int_{\Gamma_{e}} f_{i}^{s} \delta \Delta u_{i} d \Gamma_{e}
$$

Strain is splitted into linear $\left(e_{i j}\right)$ and nonlinear $\left(\eta_{i j}\right)$ strain as

$$
E_{i j}=e_{i j}+\eta_{i j}, \delta E_{i j}=\delta e_{i j}+\delta \eta_{i j}, \Delta E_{i j}=\Delta e_{i j}+\Delta \eta_{i j}, \delta \Delta E_{i j}=\delta \Delta e_{i j}+\delta \Delta \eta_{i j}
$$

The first integrand on the left-hand side of equation (9) is rewritten as

$$
\Delta E_{k l} \delta E_{i j}=\delta e_{i j} \Delta e_{k l}+\delta \eta_{i j} \Delta e_{k l}+\delta e_{i j} \Delta \eta_{k l}+\delta \eta_{i j} \Delta \eta_{k l} \approx \delta e_{i j} \Delta e_{k l}
$$

where $\Delta e=[B]\left[\Delta \tilde{u}_{e}\right],[B]$ is strain-displacement matrix. Here, we will neglect second order or nonlinear terms (underlined terms) in equation (10b).

Substituting equation (10) in equation (9), we get the following linearised equation

$$
\int_{\Omega_{e}} \delta e_{i j} C_{i j k l} \Delta e_{k l} d \Omega_{e}+\int_{\Omega_{e}} S_{i j} \delta \Delta \eta_{i j} d \Omega_{e}=\int_{\Omega_{e}} f_{i}^{b} \delta \Delta u_{i} d \Omega_{e}+\int_{\Gamma_{e}} f_{i}^{s} \delta \Delta u_{i} d \Gamma_{e}-\int_{\Omega_{e}} S_{i j} \delta e_{i j} d \Omega_{e}
$$

Corresponding equation in matrix notation for the $k^{\text {th }}$ iteration of Newton-Raphson method can be written as

$$
\begin{aligned}
\left.\left(\int_{\Omega_{e}}[B]^{T} \hat{S} d \Omega_{e}+\int_{\Omega_{e}}[G]^{T}[\tilde{S}][G] d \Omega_{e}\right)\right|_{k-1} \Delta \tilde{u}_{e}^{k} & =\int_{\Omega_{e}}[N]^{T} f^{b} d \Omega_{e} \\
& +\int_{\Gamma_{e}}[N]^{T} f^{s} d \Gamma_{e}-\left.\int_{\Omega_{e}}[B]^{T} \tilde{S} d \Omega_{e}\right|_{k-2}
\end{aligned}
$$


where $[G]$ is shape function gradient matrix, $[\tilde{S}]=\left[\begin{array}{lll}{[S]} & {[0]} & {[0]} \\ & {[S]} & {[0]} \\ \operatorname{sym} & & {[S]}\end{array}\right]$ and $[\mathbf{0}]=\left|\begin{array}{lll}0 & 0 & 0 \\ 0 & 0 & 0 \\ 0 & 0 & 0\end{array}\right|$.

Introducing the kinematic decoupling (Flory, 1961) of deformation gradient into volume preserving $\left(\mathrm{F}_{i s o}:=j^{-1 / 3} \mathrm{~F}\right)$ and volume changing $\left(\mathrm{F}_{v o l}:=J^{1 / 3} \mid\right)$ part, we can write $\mathrm{F}=\mathrm{F}_{\text {vol }}+\mathrm{F}_{i s o}$. Smoothened volumetric part of deformation gradient is given by $\bar{F}_{v o l}=\bar{J}^{1 / 3}$ l. Modified deformation gradient $(\bar{F})$ known as F-Bar is defined as the product of smoothed $\bar{F}_{v o l}$ and $F_{i s o}\left(\bar{F}=\bar{F}_{v o l} F_{i s o}\right)$. In the conventional F-Bar method, modified Green-Lagrange strain $\bar{E}=\frac{1}{2}\left(\overline{\mathrm{F}}^{T} \overline{\mathrm{F}}-I\right)$ is then directly substituted in the energy functional which makes the linearisation process complicated.

As we know, volumetric strain energy depends only on dilatational part of $\mathbf{F}\left(\mathrm{F}_{v o l}:=\right.$ $J^{1 / 3} I$ ) and locking can be removed by smoothed interpolation of $J^{-1 / 3}$ (this method hereby referred as J-Bar method).

Replacing $J$ by $\left(\bar{J}^{1 / 3}\right)^{3}$, modified volumetric strain energy is given by

$$
\Psi_{v o l}=\frac{K}{2}\left(\left(\bar{J}^{1 / 3}\right)^{3}-1\right)^{2}
$$

Minimisation of the difference between $\bar{J}^{1 / 3}$ and $J^{1 / 3}$ leads to unconstraint optimisation problem given by

$$
\min _{q \in R^{n}} R^{2}=\min _{q \in R^{n}}\left(\bar{J}^{1 / 3}-J^{1 / 3}\right)^{2}
$$

For the consistency in the definition of volumetric strain, the order of the interpolation of $\bar{J}^{1 / 3}$ within the element should be one order less than the corresponding displacement field. In line with this argument for eight noded element, $\bar{J}^{1 / 3}$ becomes constant within the element and unconstraint optimisation problem solution is given by

$$
\bar{J}^{1 / 3}=\frac{\int_{\Omega e} J^{1 / 3} d \Omega_{e}}{\int_{\Omega e} d \Omega_{e}}
$$

Similarly, for 27 noded element, $\bar{J}^{1 / 3}$ is interpolated with tri-linear shape function and unconstraint optimisation problem solution is given by

$$
\bar{J}^{1 / 3}=[\bar{N}][A]^{-1}[Z]
$$

where $[\bar{N}]=[1 \xi \eta \zeta \xi \eta \eta \zeta \zeta \xi],[A]=\int_{\Omega_{e}}[\bar{N}]^{T}[\bar{N}] d \Omega_{e}$ and $[Z]=\int_{\Omega_{e}} J^{1 / 3}[\bar{N}]^{T} d \Omega_{e}$.

After smoothening, volumetric part of second Piola-Kirchhoff stress tensor and constitutive tensor are given by 


$$
\left(S_{v o l}\right)_{i j}=\frac{\partial \Psi_{v o l}}{\partial \bar{J}^{1 / 3}} \frac{\partial \bar{J}^{1 / 3}}{\partial E_{i j}} \text { and }\left(\square_{v o l}\right)_{i j k l}=\frac{\partial\left(S_{v o l}\right)_{i j}}{\partial E_{k l}}
$$

Isochoric part of second Piola-Kirchhoff stress tensor and constitutive tensor are given by

$$
\left(S_{i s o}\right)_{i j}=\frac{\partial \Psi_{i s o}}{\partial \bar{I}_{1}} \frac{\partial \bar{I}_{1}}{\partial E_{i j}} \text { and }\left(\square_{i s o}\right)_{i j k l}=\frac{\partial\left(S_{i s o}\right)_{i j}}{\partial E_{k l}}=\frac{\partial \Psi_{i s o}}{\partial \bar{I}_{1}} \frac{\partial^{2} \bar{I}_{1}}{\partial E_{i j} \partial E_{k l}}
$$

where

$$
\frac{\partial \bar{I}_{1}}{\partial E_{i j}}=I_{3}^{-1 / 3}\left(\frac{\partial I_{1}}{\partial E_{i j}}-\frac{I_{1}}{3 I_{3}} \frac{\partial I_{3}}{\partial E_{i j}}\right)
$$

and

$$
\frac{\partial^{2} \bar{I}_{1}}{\partial E_{i j} \partial E_{k l}}=-\frac{1}{3} I_{3}^{-4 / 3}\left(\frac{\partial I_{1}}{\partial E_{i j}} \frac{\partial I_{3}}{\partial E_{k l}}+\frac{\partial I_{3}}{\partial E_{i j}} \frac{\partial I_{1}}{\partial E_{k l}}+I_{1} \frac{\partial^{2} I_{3}}{\partial E_{i j} \partial E_{k l}}-\frac{4}{3} \frac{I_{1}}{I_{3}} \frac{\partial I_{3}}{\partial E_{i j}} \frac{\partial I_{3}}{\partial E_{k l}}\right)
$$

Here, one can note that the present developed locking free 3D element represents the consistent definition of volumetric part of second Piola-Kirchhoff stress tensor and constitutive tensor. Advantage resides in the fact that the consistent representation of volumetric terms does not change the standard structure of tangent stiffness matrix and external load vector. Thus the developed element is volumetric locking free as well as simple to implement.

\section{Implementation consideration}

The numerical procedure to implement the standard method and proposed J-Bar method is briefly described as:

1 Call elemental nodal displacements $\left(\left(\tilde{u}_{e}\right)_{n}\right)$ from the previous load step.

2 Compute $E, I_{1}, I_{2}, J, \bar{I}_{1}$ and $\bar{I}_{2}$.

3 For standard method, compute total stress $(S)$ and constitutive tensor ( $\square$ ) using equation (4) and equation (5), respectively.

4 For J-Bar method, compute $\bar{J}^{1 / 3}$ using equation (15) or equation (16) as per the type of element.

a compute $S_{v o l}$ and $\square$ vol using equation (17)

b compute $S_{\text {iso }}$ and $\square$ iso using equation (18)

c compute total stress and constitutive tensors.

$$
S=S_{v o l}+S_{i s o} \text { and } \square=\square \text { vol }+\square \text { iso }
$$

5 Compute the global internal and external load vectors to solve system of algebraic equation. 
6 If convergence is achieved then update nodal displacement.

$$
\left(\tilde{u}_{e}\right)_{n+1}=\left(\tilde{u}_{e}\right)_{n}+\Delta \tilde{u}
$$

7 Apply load increment for the next step and go to step 1.

Based on the above formulation, a FORTRAN ${ }^{\mathrm{TM}}$ code is developed in-house and validated with the results available based on the B-Bar element.

\section{Numerical examples}

In this section, examples are considered to demonstrate the volumetric locking in isotropic hyperelastic solids and robustness of the proposed locking free scheme.

\subsection{Performance of higher order element in nearly incompressible medium}

Tri-linear J-Bar formulation is validated by considering the finite stretching of neo-Hookean rubber block $(2 \mathrm{~m} \times 1 \mathrm{~m} \times 1 \mathrm{~m})$ which is fixed at one end and subjected to deformation independent uniformly distributed tensile load at the other end. Material parameters for the neo-Hookean material model are $K=29,980 \mathrm{kPa}$ and $C_{1}=60 \mathrm{kPa}$ (Foster and Nejad, 2015).

The displacement of point A obtained through the finite element discretisation of the block with the 27 noded hexahedral elements is shown in Figure 1. It can be seen that the standard method is showing slightly stiffening behaviour whereas tri-linear J-Bar is predicting the locking free response consistent with the Foster and Nejad (2015). It is also found that with the $8 \times 4 \times 4$ mesh of 27 noded element using standard method, volumetric locking is eliminated.

Figure 1 Convergence of tip displacement with mesh refinement of 27 noded elements (see online version for colours)

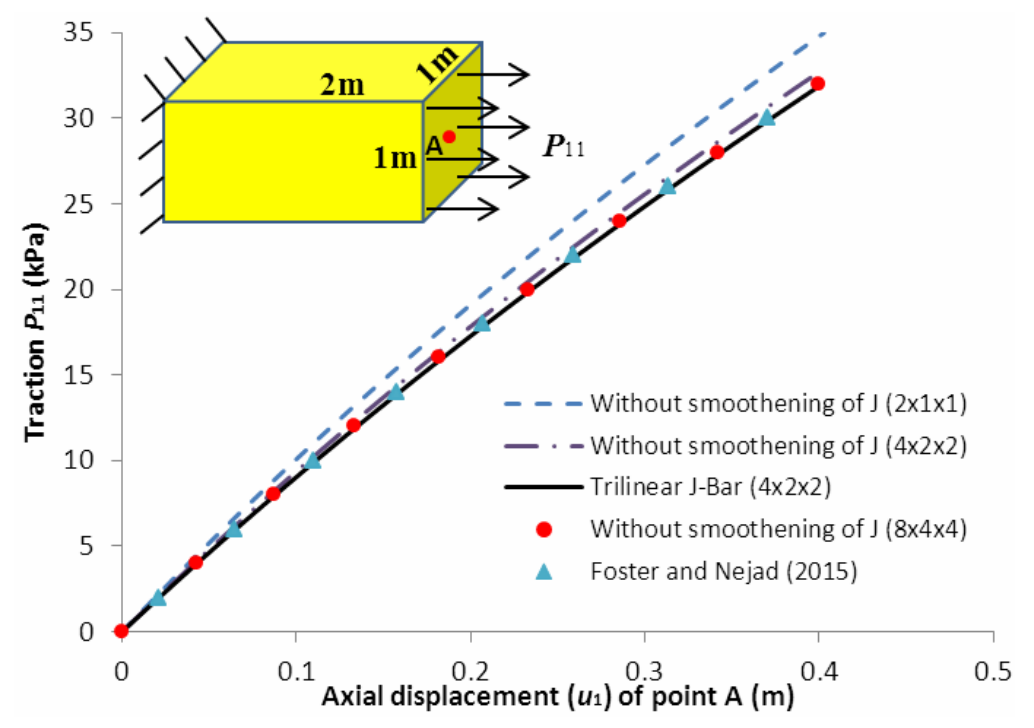


Figure 2 Deformed shape of isotropic neo-Hookean block undergoing large deformation under uniform tensile load of $150 \mathrm{kPa}$ (see online version for colours)

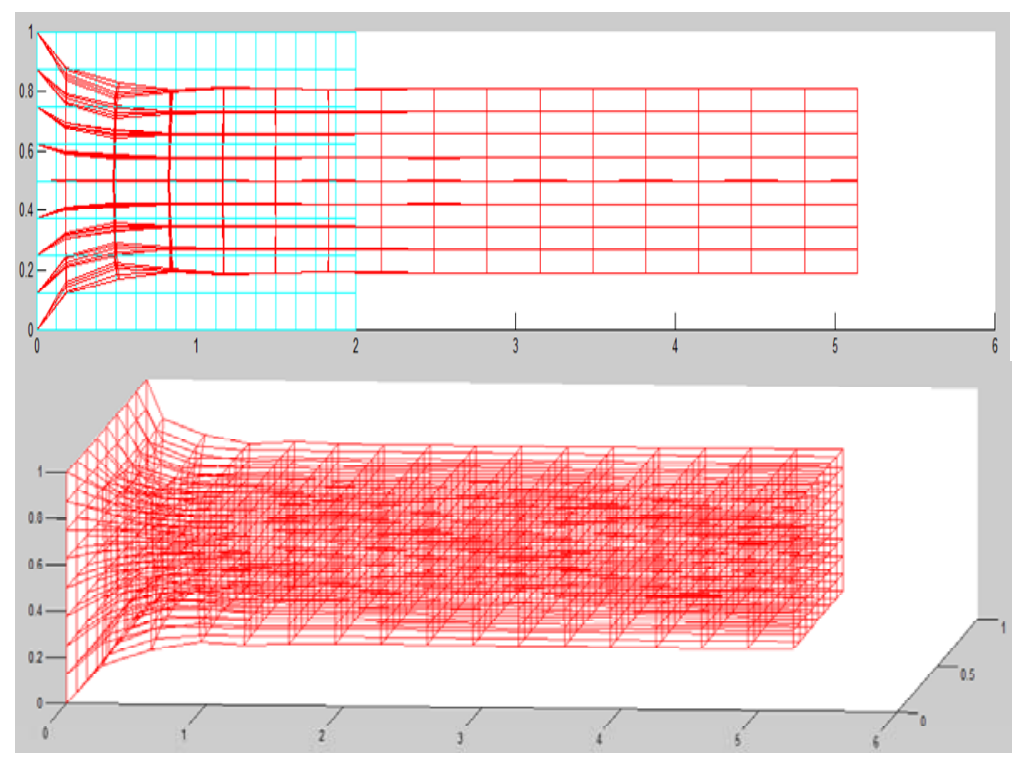

Note: cyan: undeformed, red: deformed.

Next, the finite deformation of the rubber block stretched up to 2.5 times of its undeformed length is studied. The standard method is used with $8 \times 4 \times 4$ (=128 elements) discretisation to simulate the deformed shape shown in Figure 2 for the uniformly distributed tensile load of $150 \mathrm{kPa}$. The undeformed and deformed shapes of the rubber block are shown by cyan and red colour, respectively.

\subsection{Lower order element for modelling of nearly incompressible solids}

Based on the results presented using 27 noded brick element, one can conclude that there is no significant difference in the displacement field with and without J-Bar approach. This is due to the superior performance of 27 noded hexahedral elements even in nearly incompressible regime. However, it is computationally expensive so it is preferable to use lower order brick elements (e.g., eight noded brick element).

\subsubsection{Finite stretching of neo-Hookean solid}

In this example, the displacement of point $\mathrm{A}$ obtained by discretising the block with $8 \times 4 \times 4$ elements using eight noded hexahedral element is shown in Figure 3 . It can be seen that the standard formulation without smoothening of $J$ is showing stiff behaviour whereas J-Bar-based element eliminates the volumetric locking and the results match with Foster and Nejad (2015) B-Bar-based element. 
Figure 3 Traction $\left(P_{11}\right)$ versus axial displacement $\left(u_{1}\right)$ curves for neo-Hookean rubber (see online version for colours)

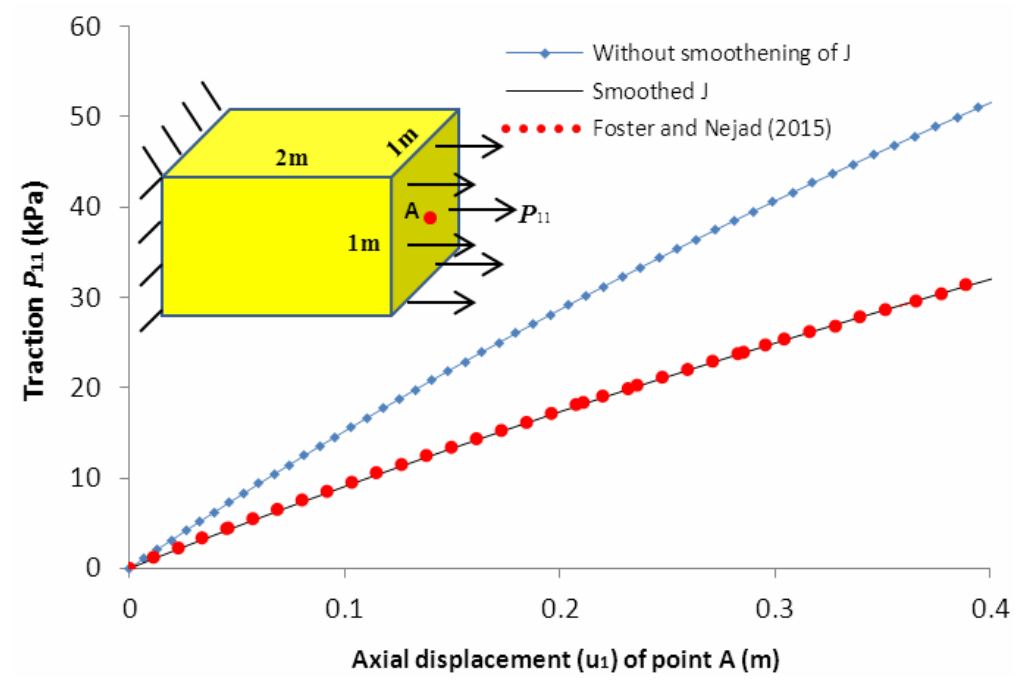

Figure 4 Convergence of transverse displacement of point A $\left(u_{1}\right)$ on mesh refinement (see online version for colours)

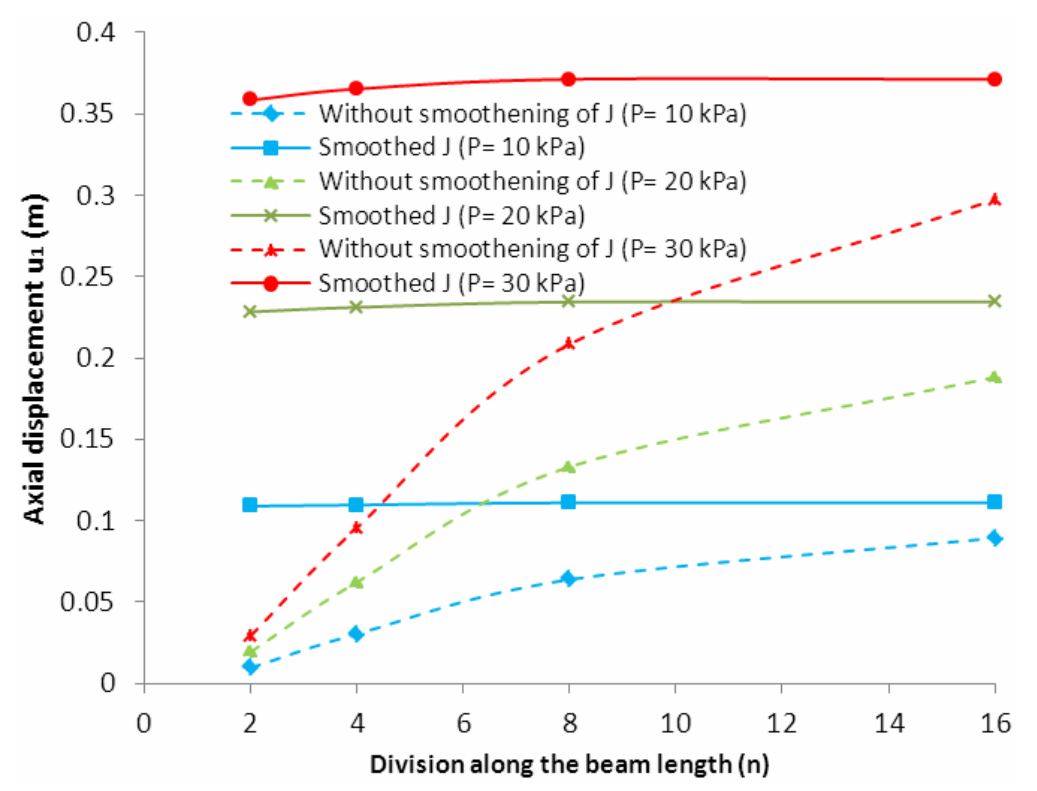

Now, we investigate the effect of mesh refinement on the accuracy of tip displacement of point A. Domain is discretised with $n \times \frac{n}{2} \times \frac{n}{2}$ mesh of eight noded brick element where $n=2,4,8,16$ is the number of divisions along the length of the block. Convergence study is shown for three loads viz., $P_{11}=10 \mathrm{kPa}, P_{11}=20 \mathrm{kPa}$ and $P_{11}=30 \mathrm{kPa}$. It can be observed that element based on the smoothening of $J$ is performing better and producing 
realistic displacement (see Figure 4). Contrary to this, standard element depicts locking and under-predicts the displacement. It may also be concluded from Figure 4 that the block under axial loading depicts softening nonlinearity leading to the increase in the incremental displacement with the increase in the axial load.

\subsubsection{Finite deformation of $3 D$ cantilever beam under bending load}

In this section, we investigate the three-dimensional bending deformation of neo-Hookean cantilever rubber beam subjected to deformation independent uniformly distributed transverse load $\left(P_{13}\right)$ at the free end. Material parameters and discretisation are same as that used in previous example. The vertical tip displacement of point A versus load $\left(P_{13}\right)$ curves obtained using the elements with and without smoothening of $J$ are compared in Figure 5. It can be seen that the element without the smoothening of $\mathrm{J}$ is under predicting the displacement (locking behaviour) whereas smoothed J-based element is volumetric locking free.

Figure 5 Traction $\left(P_{13}\right)$ versus transverse displacement $\left(u_{3}\right)$ curves for neo-Hookean rubber (see online version for colours)

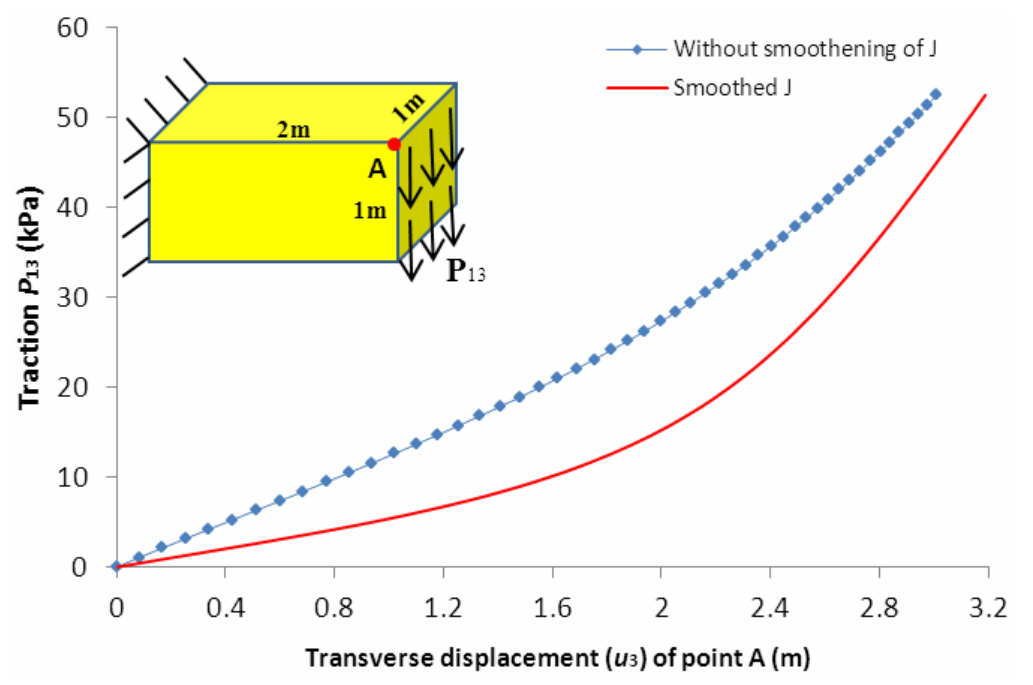

Finally, the convergence of the transverse displacement of point ' $A$ ' of the neo-Hookean rubber beam with the mesh refinement is studied. Three values of uniformly distributed transverse load are considered in the analysis viz., $P_{13}=10 \mathrm{kPa}, P_{13}=20 \mathrm{kPa}$ and $P_{13}=30 \mathrm{kPa}$.

Mesh convergence study is performed using $n \times \frac{n}{2} \times \frac{n}{2}$ discretisation, where $n=2,4$, 8,16 is the number of divisions along the length of the beam. It can be observed that irrespective of load intensity, proposed J-Bar-based element performs quite well whereas standard element without the smoothening of $J$ shows over stiffening behaviour (see Figure 6). It can also be inferred from Figure 6 that the block under bending depicts hardening nonlinearity leading to the decrease in the rate of displacement increase with the increase in the transverse load. 
Figure 6 Convergence of transverse displacement of point A $\left(u_{3}\right)$ on mesh refinement (see online version for colours)

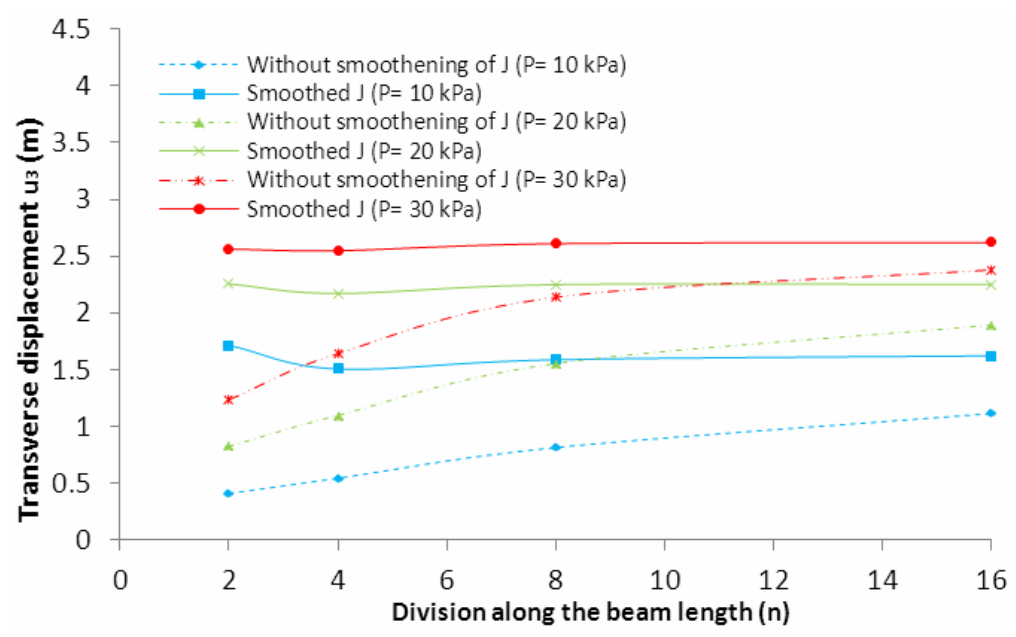

It is apt to mention here that the computational time per iteration for the same number of elements is nearly the same for the proposed $J$ bar and conventional formulations. However, total number of iterations per load step increases in former case leading to the increase in the computational time. However, the $J$ bar approach gives converged results with significantly smaller number of elements making it computationally efficient.

\section{Concluding remarks}

In this work, we have demonstrated the superior performance of the developed eight noded 3D solid elements with the smoothening of $J$ by considering the axial stretch and bending of a neo-Hookean solid. We remark that the developed method presents the consistent representation of volumetric terms which in turn preserves the standard structure of elemental stiffness matrices and load vectors. A comparative study is performed considering higher and lower order elements for modelling incompressible solids. In future, the developed method can be extended to study the visco-hyperelastic response of nearly incompressible isotropic as well as anisotropic solids.

Based on the numerical simulations, the following conclusions can be drawn:

1 Higher order element performs better even in nearly incompressible medium with elimination of locking with refinement.

2 Results predicted by developed J-Bar-based element are consistent with B-Bar method with the simplicity of implementation and computational efficiency.

3 Irrespective of deformation mode, standard element formulation (lower order elements) exhibits locking which remains significant even with mesh refinement. 


\section{References}

Akhrass, D.A., Drapier, S., Bruchon, J. and Fayolle, S. (2012) 'Stabilized finite element methods to deal with incompressibility in solid mechanics in finite strains', edited by Eberhardsteiner, J. et al. (Eds.): European Congress on Computational Methods in Applied Sciences and Engineering (ECCOMAS 2012), Vienna, Austria.

Bernardin, A.O., Hale, J.S. and Cryon, C.J. (2015a) 'Volume-averaged nodal projection method for nearly-incompressible elasticity using meshfree and bubble basis functions', Computer Methods in Applied Mechanics and Engineering, Vol. 285, pp.427-451.

Bernardin, A.O., Puso, M.A. and Sukumar, N. (2015b) 'Improved robustness for nearly-incompressible large deformation meshfree simulations on Delaunay tessellations', Computer Methods in Applied Mechanics and Engineering, Vol. 293, pp.348-374.

Bertram, A. (2007) Elasticity and Plasticity of Large Deformations, 3rd ed., pp.150-152, Springer, Berlin.

Boyce, M.C. and Arruda, E.M. (2000) 'Constitutive models of rubber elasticity: a review', Rubber Chemistry and Technology, Vol. 73, No. 3, pp.504-523.

de Souza Neto, E.A., Peric, D., Dutko, M. and Owen, D.R.J. (1996) 'Design of simple low order finite elements for large strain analysis of nearly incompressible solids', International Journal of Solids and Structures, Vol. 33, Nos. 20-22, pp.3277-96.

Duong, M.T., Nguyen, N.H. and Staat, M. (2015) 'Physical response of hyperelastic models for composite materials and soft tissues', Asia Pacific Journal on Computational Engineering, Vol. 2, No. 3, pp.1-18.

Elguedj, T., Bazilevs, Y., Calo, V.M. and Hughes, T.J.R. (2008a) ' $\overline{\mathrm{F}}$ projection methods for finite deformation elasticity and plasticity using NURBS-based isogeometric analysis', International Journal of Material Forming, Vol. 1, No. 1, pp.1091-1094.

Elguedj, T., Bazilevs, Y., Calo, V.M. and Hughes, T.J.R. (2008b) ' $\bar{B}$ and $\overline{\mathrm{F}}$ projection methods for nearly incompressible linear and nonlinear elasticity and plasticity using higher-order NURBS elements', Computer Methods in Applied Mechanics and Engineering, Vol. 197, Nos. 33-40, pp.2732-2762.

Flory, P.J. (1961) 'Thermodynamic relations for high elastic materials', Trans. Faraday Society, Vol. 57, pp.829-838.

Foster, C.G. and Nejad, T.M. (2015) 'Trilinear hexahedral with integral-averaged volumes for nearly incompressible nonlinear deformation', Scientific Research Publishing, Engineering, Vol. 7, pp.765-788.

Fried, I. (1974) 'Finite element analysis of incompressible material by residual energy balancing', International Journal of Solids and Structures, Vol. 10, No. 9, pp.993-1002.

Guo, Z. and Sluys, L.J. (2006) 'Application of new constitutive model for the description of rubber-like materials under monotonic loading', International Journal of Solids and Structures, Vol. 43, No. 9, pp.2799-2819.

Holzapfel, G.A. (1996) 'On large strain viscoelasticity: continuum formulation and finite element applications to elastomeric structures', International Journal for Numerical Methods in Engineering, Vol. 39, No. 22, pp.3903-3926.

Hughes, T.J.R. (1980) 'Generalization of selective integration procedures to anisotropic and nonlinear media', International Journal for Numerical Methods in Engineering, Vol. 15, No. 9, pp.1413-18.

Hughes, T.J.R. (1987) The Finite Element Method: Linear Static and Dynamic Finite Element Analysis, pp.240-242, Prentice-Hall, Englewood Cliffs, New Jersey.

Liu, X., Liu, F., Han, L., Liu, R. and Li, K. (2016) 'A CAD/CAE integration method based on excel', International Journal of Material and Structural Integrity, Vol. 10, No. 4, pp.181-194. 
Malkus, D.S. and Hughes, T.J.R. (1978) 'Mixed finite element methods-reduced and selective integration techniques: a unification of concepts', Computer Methods in Applied Mechanics and Engineering, Vol. 15, No. 1, pp.63-81.

Nagtegaal, J.C., Parks, D.M. and Rice, J.R. (1974) 'On numerically accurate finite element solutions in the fully plastic range', Computer Methods in Applied Mechanics and Engineering, Vol. 4, No. 2, pp.153-77.

Schröder, J. and Neff, P. (2003) 'Invariant formulation of hyperelastic transverse isotropy based on polyconvex free energy functions', International Journal of Solids and Structures, Vol. 40, No. 2, pp.401-445.

Simo, J.C., Taylor, R.L. and Pister, K.S. (1985)' Variational and projection methods for the volume constraint in finite deformation elasto-plasticity', Computer Methods in Applied Mechanics and Engineering, Vol. 51, Nos. 1-3, pp.177-208.

Suzuki, J.L. and Bittencourt, M.L. (2016) Computational Modelling, Optimization and Manufacturing Simulation of Advanced Engineering Materials, pp.113-150, Springer, Switzerland.

Weiss, J.A., Maker, B.N. and Govindjee, S. (1996) 'Finite element implementation of incompressible, transversely isotropic hyperelasticity', Computer Methods in Applied Mechanics and Engineering, Vol. 135, Nos. 1-2, pp.107-128.

Wriggers, P. and Carstensen, C. (2009) 'Mixed finite element technologies', CIMS Courses and Lectures, Vol. 509, pp.160-163, Springer, Wien. 